\title{
Entretien
}

\section{L'entomologie médicale : de la recherche fondamentale à la lutte antivectorielle}

\section{Propos recueillis par Daniel Bley et Danièle Magda}

\author{
Didier Fontenille ${ }^{1}$, Daniel Bley², Danièle Magda ${ }^{3}$ \\ 1 Entomologiste médical, IRD, UR016 CCPV, Centre IRD de Monpellier, 911 avenue Agropolis, BP 64501, \\ 34394 Montpellier cedex 5, France \\ 2 Anthropologue, CNRS, UMR6012 ESPACE, DESMID, Université de la Méditerranée Aix-Marseille 2, \\ Faculté des sciences de Luminy, 163 avenue de Luminy, 13288 Marseille cedex 09, France \\ 3 Écologue, INRA, UMR AGIR, Chemin de Borde Rouge, Auzeville, 31320 Castanet Tolosan, France
}

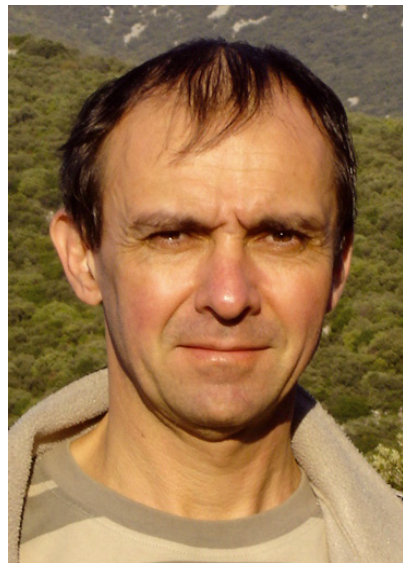

Éléments de biographie

Didier Fontenille, directeur de recherche à l'Institut de recherche pour le développement (IRD), est entomologiste médical, spécialiste de la biologie, de la génétique et du contrôle des insectes vecteurs de maladies comme le paludisme, la dengue ou le Chikungunya. Après dix-huit ans passés à traquer les moustiques à l'institut Pasteur de Madagascar, puis à l'institut Pasteur de Dakar au Sénégal et à l'Organisation de coordination pour la lutte contre les endémies en Afrique centrale (OCEAC) au Cameroun, il est maintenant basé à Montpellier. Depuis 2005, il dirige l'unité de recherche IRD Caractérisation et contrôle des populations de vecteurs (CCPV), forte de soixante scientifiques basés en Amérique du Sud, en Afrique et en Europe. Expert d'organismes nationaux et internationaux, il est responsable de plusieurs programmes de recherche sur les vecteurs de virus et de parasites.

NSS : Les maladies vectorielles transmissibles, tel le paludisme, qui affectent depuis toujours les pays du Sud peuvent aujourd'hui, avec les changements climatiques et la recrudescence des mobilités humaines et animales, menacer les zones tempérées. Il s'agit là de questions scientifiques, situées à l'interface des champs de l'environnement et de la santé, qui permettent de s'interroger sur les approches interdisciplinaires, sur les relations entre experts et acteurs, et qui posent de façon plus générale la question de la relation entre science et société. Nous aimerions vous interroger sur la place qu'occupe

Auteur correspondant : D. Bley, danielbley@aol.com l'entomologie médicale dans ce contexte et sur les évolutions qu'a connues cette discipline. Par ailleurs, l'IRD a fait appel à vous pour diriger une importante expertise collégiale commanditée par quatre ministères concernant la lutte antivectorielle en France (Encadré). Pouvezvous, d'abord, nous dire quels en étaient les attendus et à quelles conclusions vous aboutissez ?

Didier Fontenille : Cette expertise a été décidée suite à l'augmentation des épisodes de maladies, humaines et animales, liées à une transmission vectorielle en France, en particulier dans ses régions et communautés d'outremer, au risque lié à l'expansion de vecteurs d'agents pathogènes et à la difficulté d'y faire face avec le système 


\begin{abstract}
Encadré. Conclusions de l'expertise collective sur la lutte antivectorielle en France ${ }^{1}$
Les maladies vectorielles transmissibles ont la particularité d'être transmises par un vecteur, un arthropode hématophage (moustiques et tiques en majorité), qui assure la transmission biologique active d'un agent pathogène (virus, bactérie, parasite). Elles peuvent affecter l'homme (Chikungunya, paludisme, dengue, maladie de Lyme...) ou les animaux (fièvre catarrhale ovine, trypanosomose en Guyane...). L'expertise collégiale portant sur la lutte antivectorielle en France (LAV) a été produite par un collège d'experts issus de l'ensemble des institutions de recherche impliquées par le sujet. À l'issue d'une année de travail, ce collège a produit un état des lieux complet dans toutes ses composantes disciplinaires et une série de huit recommandations prioritaires d'ordre opérationnel :
\end{abstract}

- Créer un centre national d'expertise sur les vecteurs et le risque vectoriel (CNEV)

Le CNEV devrait devenir l'outil de pilotage de la lutte antivectorielle en France, avec un rôle dans l'expertise, l'évaluation, l'aide à la décision, la formation, le conseil, l'échange d'informations et l'incitation à la recherche, aux niveaux français, européen et circumméditerranéen.

\title{
- Redéfinir le cadre juridique et la gouvernance
}

La prévention des maladies à transmission vectorielle est une question de santé publique relevant de la compétence de l'État. Il est proposé de créer un dispositif législatif unique pour encadrer la lutte contre les vecteurs en distinguant bien prévention et mise en œuvre de la lutte antivectorielle. Celle-ci serait intégrée dans les missions des futures agences régionales de santé (ARS) et des outils juridiques de coopération seraient proposés pour associer les collectivités territoriales.

\section{- Préciser les stratégies d'intervention}

Les stratégies d'intervention dans le cadre d'épidémies ou de luttes antivectorielles de routine méritent d'être mieux définies. Il est proposé de rédiger des plans d'action, sur le modèle West Nile, sous la responsabilité de l'État et avec la contribution du CNEV.

\section{- Évaluer les interventions}

Des lacunes importantes ont été notées dans l'évaluation des actions opérationnelles, l'évaluation économique et l'évaluation des effets non intentionnels. Il conviendrait d'initier un travail sur les indicateurs, puis d'instiller une culture de l'évaluation dans les services.

\section{- Soutenir la recherche}

Des recherches sont à développer prioritairement dans trois domaines : connaissance des mécanismes fins de la transmission vectorielle ; évaluation du risque entomologique, en particulier dans le cadre des changements climatiques et environnementaux ; stratégies de contrôle de la transmission moins polluantes, plus ciblées et plus durables.

\section{- Améliorer la communication}

Des actions ciblées selon les contextes et les groupes sociaux sont nécessaires. L'INPES² pourrait jouer un rôle fédérateur.

\section{- Améliorer la formation}

L'amélioration du dispositif de lutte nécessite une meilleure formation théorique et pratique des cadres de santé publique et vétérinaire et des agents techniques. Les formations pourront être proposées par le CNFPT ${ }^{3}$, les réseaux professionnels de type ADEGE, des universités, l'EHESP ou le CNEV.

\section{- Sortir les services de leur isolement}

Le développement de réseaux régionaux et internationaux sera fortement appuyé afin d'améliorer la surveillance des vecteurs et la réponse opérationnelle.

actuel. C'est par exemple le cas des épidémies récentes de Chikungunya dans l'océan Indien, de la dengue dans les départements français des Amériques, de la fièvre

\footnotetext{
${ }^{1}$ Ces éléments sont issus du dossier réalisé par le département Expertise et Valorisation avec l'aide de la Délégation à l'information et à la communication de l'IRD (contact : dev@ird.fr) pour la publication de l'ouvrage dirigé par D. Fontenille et al., La Lutte antivectorielle en France, Marseille, IRD Éditions, coll. «Expertise collégiale», 2009, 536 p. + CD-ROM.

2 INPES : Institut national de prévention et d'éducation pour la santé.

${ }^{3}$ CNFPT : Centre national de la fonction publique territoriale; ADEGE : Assistance au développement et à la gestion des entreprises; EHESP : École des hautes études en santé publique.
}

catarrhale ovine en métropole, d'épisodes de fièvres de la vallée du Rift à Mayotte, d'encéphalites à tiques en Alsace, de la maladie de Chagas en Guyane, ou de l'expansion d'Aedes albopictus dans le Sud de la métropole. Ces problèmes de santé publique humaine ou vétérinaire nécessitent des réponses coordonnées et adaptées, que les différents services et opérateurs ne peuvent actuellement pas fournir de manière optimale.

Le groupe d'experts a donc réalisé un état des lieux de la lutte antivectorielle en France dans toutes ses dimensions : juridique, gestion de crise, différentes stratégies de lutte, évaluation économique des activités opérationnelles et des effets indirects sur l'environnement, formation, état de la recherche et des collaborations régionales et internationales. Huit recommandations majeures 
ont été proposées aux ministères commanditaires, la recommandation la plus importante étant la création d'un Centre national d'expertise sur les vecteurs et le risque vectoriel (CNEV), sur lequel pourraient s'appuyer les opérateurs, les régions et l'État. Ce centre pourrait contribuer à éclairer la politique nationale de lutte antivectorielle et la recherche opérationnelle et serait un acteur important de la formation. Il s'agirait d'une structure d'expertise multidisciplinaire dans laquelle seraient mobilisées des compétences bien entendu en entomologie médicale, qui est ma discipline, mais aussi en environnement, en santé publique et vétérinaire, en sciences sociales.

NSS : Quel itinéraire vous a conduit à devenir un spécialiste de l'entomologie médicale?

Didier Fontenille : Je suis un pur naturaliste, un pur produit de l'Université. À l'origine, je suis professeur de sciences naturelles. C'est d'ailleurs un grand atout pour faire de l'entomologie médicale ou vétérinaire. Ma formation me permet de reconnaître la nature des sols créant des gîtes larvaires à moustiques, et de m'intéresser aussi aux autres espèces de l'écosystème, comme les reptiles, les oiseaux ou d'autres arthropodes ; elle me permet donc d'avoir une vision assez systémique d'un sujet. Par ailleurs, j'avais aussi un intérêt particulier pour la recherche pour le développement, spécialement au bénéfice des pays du Sud. Comment être utile aux pays du Sud ou aux pays en voie de développement et en même temps satisfaire un intérêt pour la nature, les écosystèmes et l'entomologie? Finalement, j'ai trouvé une interface avec l'entomologie médicale. Comme il n'y avait plus à mon époque de formation qui soit spécifique en entomologie médicale, je me suis formé au contact de professionnels plus âgés que moi à l'Institut Pasteur et à l'ORSTOM. J'ai ensuite compris que, pour étudier la transmission d'agents pathogènes par les vecteurs, il fallait aborder les questions génétiques. Je n'étais pas au départ un spécialiste de cette discipline, même si ma formation m'avait donné des bases assez solides. J'ai donc approfondi ce domaine et, surtout, j'ai cherché des étudiants plus compétents que moi pour développer ces aspects-là dans mon unité de recherche, ce qu'ils font aujourd'hui. J'ai par ailleurs suivi des cours de virologie à l'Institut Pasteur et je pense donc avoir une vision assez large.

\section{Une discipline au milieu du gué...}

NSS : Malgré la centralité que semble présenter votre discipline dans les conclusions de l'expertise sur la lutte antivectorielle, un article de Dominique Cuisance publié en $2003^{4}$ faisait pourtant état de la diminution des entomologistes médicaux.

\footnotetext{
4 Cuisance, D., 2003. Entomologie médicale et vétérinaire en France : regard sur une situation, Insectes, 131, 25-27.
}

Didier Fontenille : Dominique Cuisance, associé à un certain nombre d'autres chercheurs, dont moi pour l'IRD, était en effet arrivé au constat du manque de relève en jeunes entomologistes en France métropolitaine, dans les départements et territoires d'outre-mer et, plus largement, dans les pays où la France intervient, soit spontanément, soit à la demande de nos pays partenaires. Nous avons communiqué sur les résultats de cette enquête dans les ministères et dans le cadre d'un colloque à Montpellier, et j'ai l'impression depuis que la situation s'est améliorée. Il y a eu une prise de conscience de l'importance du sujet en France et, plus largement, au niveau de l'Europe, et ensuite, une volonté des instituts de recherche et en particulier de l'IRD de soutenir des recherches sur les conséquences du changement climatique sur les maladies à transmission vectorielle. Et puis, bien sûr, il y a eu l'épidémie de Chikungunya en 2006 à l'île de la Réunion...

Nous avons, par exemple, créé un master international d'entomologie médicale. Il y a donc eu un certain nombre de choses qui se sont mises en place et dont il est possible de voir maintenant les retombées, depuis un an ou deux. Je crois que, si cette enquête était refaite aujour$\mathrm{d}$ 'hui, on pourrait constater une reprise des recrutements dans ce domaine de compétence, que ce soit au Cirad, à l'IRD, dans les instituts Pasteur ou dans les agences opérationnelles du type Entente interdépartementale pour la démoustication (EID).

NSS : Mais est-ce que l'entomologie médicale en tant que discipline n'a pas néanmoins perdu de son importance?

Didier Fontenille : D'abord, je parlerai d'entomologie médicale et vétérinaire, parce qu'il ne faut pas séparer les deux. La plupart des agents pathogènes qui sont transmis à l'homme sont aussi des pathogènes animaux. En fait, c'est la thématique même de l'entomologie médicale qui a beaucoup changé. Avant, c'était une science essentiellement descriptive; et puis, avec l'évolution de la recherche, elle s'est orientée vers des aspects plus conceptuels. Du coup, les sciences qui pouvaient être des sciences descriptives avec des visées explicatives sont tombées un peu en désuétude. Il n'y a plus eu d'étudiants intéressés pour faire de la systématique en entomologie, ni pour faire de l'épidémiologie incluant une composante entomologique. Ce qui attirait les étudiants, dans les dix dernières années, c'était la biologie intégrative ou la génomique évolutive. Et, dans ces derniers cas, on considérait l'insecte vecteur de maladies non pas comme sujet d'étude, comme le faisaient les anciens entomologistes médicaux, mais comme objet d'étude au même titre qu'une drosophile ou qu'une boîte de Pétri.

NSS : Votre dernière remarque nous amène à nous interroger pour savoir si, dans le monde de l'entomologie médicale actuel, il n'y aurait pas une séparation entre 
ceux qui travaillent des molécules en laboratoire et ceux qui travaillent sur l'écologie du vecteur sur le terrain.

Didier Fontenille : Il y a eu une dichotomie, une divergence, à un moment et de nouveau il y a intégration. La nouvelle génération d'entomologistes médicaux est censée maîtriser les aspects systématiques, l'écologie, l'épidémiologie, mais en utilisant des outils dits modernes de biologie moléculaire, de génomique, de systèmes d'information géographique, voire de bioinformatique.

Je pense qu'on a su former des jeunes avec de nouvelles compétences, simplement en leur permettant d'intégrer ces nouvelles disciplines, ces nouveaux concepts dans des thématiques finalisées. L'entomologie médicale est une science qui peut être très fondamentale tout en étant finalisée, puisque l'objectif final est d'arriver à diminuer le poids de la maladie, animale ou humaine ; et, à ce titre, elle s'inscrit dans le domaine de la santé publique.

NSS : Nous souhaiterions aussi savoir comment s'est fait le lien entre l'entomologie médicale et les domaines de l'écologie.

Didier Fontenille : Jusqu'à ces dernières années, les aspects génétiques étaient mobilisés en relation avec l'agent pathogène lui-même, et, quand j'ai abordé dans ma formation les aspects génétiques, c'était plus en collaboration avec des parasitologues, des virologues, etc. Mais un agent pathogène et même un virus font aussi partie de l'écosystème. Petit à petit, nous avons donc réintroduit dans notre unité, en regard de ce qui se passait dans la communauté scientifique internationale, des aspects de génétique environnementale. Par exemple, aujourd'hui nous travaillons sur les gènes impliqués dans l'adaptation des moustiques à différents biotopes, différents environnements, et nous examinons en particulier quels sont les mécanismes génétiques mobilisés par les moustiques pour s'adapter à ces changements environnementaux.

NSS : Comment procédez-vous pour mettre en place ce type de recherches?

Didier Fontenille : L'entomologie a eu une phase très descriptive, très spécialisée, mais nous en venons à des visions beaucoup plus larges qui nécessitent le développement de programmes pluridisciplinaires avec des géographes, des généticiens, des collègues spécialisés dans les systèmes d'information géographique, des anthropologues... Finalement, nous essayons de construire différentes couches d'un système d'information géographique permettant d'analyser la répartition de certains gènes ou de leur fréquence. Je pense qu'il y a peu d'équipes au monde qui sont allées dans les différents niveaux d'analyse jusqu'aux gènes. En général on s'arrête à l'espèce, voire à la population; mais, dans notre équipe, nous considérons que, pour certains vecteurs, l'unité finale c'est le gène.
Par exemple, un étudiant, qui a soutenu sa thèse récemment, s'est intéressé à la distribution au Cameroun d'une espèce de moustique vecteur du paludisme, Anopheles funestus, en fonction de critères environnementaux et climatiques. Dans la distribution de cette espèce, ce qui est important à regarder, c'est la distribution des différentes populations. Nous disposons pour cela d'un marqueur génétique des populations : ce sont les inversions chromosomiques, qui sont observables au microscope; nous regardons la distribution de la nature et de la fréquence de ces inversions chromosomiques sur des transects nord-sud, qui vont de la forêt équatoriale jusqu'aux zones très sèches sahéliennes. Nous mettons ensuite ces distributions en relation avec un certain nombre de variables environnementales et anthropiques, comme le tracé des routes, la distribution des villages, etc. Et si les milieux changent, les populations changent : c'est ça qui est incroyable. Par exemple, nous avons montré que, dans l'hypothèse de changements climatiques, l'espèce ne disparaîtra pas, mais s'adaptera en mobilisant des gènes différents. Ce sont donc les fréquences génétiques qui seront différentes, avec la sélection de gènes adaptés à ces nouveaux environnements. Ce résultat est très important, et démontre qu'il ne faut pas raisonner au niveau des espèces, mais des populations.

\section{... à la recherche d'un dialogue interdisciplinaire}

NSS : Dans les programmes pluridisciplinaires auxquels vous avez fait référence, quelle est la place des entomologistes?

Didier Fontenille : Nous travaillons en équipe. Par exemple, l'étudiant dont je viens de parler a fait sa thèse en développant davantage les aspects biologiques et génétiques du vecteur. Mais, dans l'équipe, idéalement il faut avoir un écologue capable d'identifier les variables du milieu, un géographe qui connaît suffisamment les variables climatiques et un anthropologue lorsqu'il y a des composantes anthropiques fortes. Parfois, il faut aussi des spécialistes en génétique, et même des spécialistes de modèles en génétique. Et puis, on a mis en évidence des choses complexes. Par exemple, ce travail nous a permis de réfléchir sur les mécanismes de spéciations, et c'est alors de la génétique très conceptuelle.

En fait, l'entomologiste retrouve sa place assez facilement, tout dépend de qui pose la question et quel est l'objectif final. Par exemple, dans l'unité de recherche que je dirige, et en simplifiant, notre interrogation essentielle de recherche est : "Qu'est-ce que c'est qu'un vecteur? »Il faut tout de même savoir qu'il y a plusieurs millions d'espèces d'insectes sur terre, dont 3500 de moustiques, et qu'il y a seulement 10 espèces de moustiques qui transmettent la dengue à l'homme. Il faut donc 
expliquer pourquoi il y en a 3490 qui ne transmettent pas cette maladie, et c'est ça la vraie question. Car une fois que nous savons pourquoi une espèce est vectrice dans un environnement donné, pourquoi elle pique tel animal, pourquoi elle est capable de répliquer tel parasite, comment elle s'adapte, etc., alors on va pouvoir mieux la combattre, utiliser des outils appropriés, ne pas se tromper de cible... C'est pour cette raison que nous avons mobilisé autour de nous des équipes pluridisciplinaires.

Mais ce peut être aussi un géographe qui s'intéresse à l'environnement global dont le moustique est une des composantes et qui peut nous solliciter pour travailler sur les moustiques. Ce peut être aussi un anthropologue ou un sociologue qui développe un programme centré sur les comportements humains, et l'entomologie médicale sera alors une des composantes de ce programme. Quand la question finale porte sur la lutte antivectorielle, le pivot, c'est l'entomologiste.

NSS : Et qu'en est-il des collaborations avec les médecins?

Didier Fontenille : Quand on fait de l'entomologie médicale, il y a tout de même le mot médical, car l'objectif est de diminuer le poids de la maladie chez l'homme ou l'animal. Donc, pour nous, il n'y a pas d'ambiguïté, nous travaillons dans le domaine de la santé publique. Nous intégrons complètement la composante médicale et les relations sont plutôt bonnes avec les médecins. Il y a deux niveaux d'implication. Le premier niveau concerne la recherche : nous essayons de démêler les différentes composantes de ce que nous, entomologistes, nous appelons un système vectoriel, qui inclut l'homme. Donc, pour la composante humaine, il y a bien sûr des médecins, des cliniciens et des immunologistes. Le médecin va parler d'une maladie et ne centre pas tout à fait le système pathogène de la même manière qu'un entomologiste médical, mais nous partageons une vision sur l'ensemble des composantes. Le deuxième niveau d'implication, c'est la lutte opérationnelle contre la maladie. Le médecin a besoin qu'on prenne en charge la lutte antivectorielle. C'est la deuxième étape de l'entomologie médicale, la partie finalisée : par la lutte antivectorielle, il nous faut participer à la diminution de l'incidence de la maladie et de sa prévalence.

NSS : Vous nous aviez fait remarquer, au début de notre entretien, que l'entomologie était médicale mais aussi vétérinaire, et pourtant vous ne citez pas les vétérinaires.

Didier Fontenille : Jusqu'à présent, les vétérinaires en France n'ont pas vraiment été confrontés à des épizooties, c'est-à-dire des épidémies animales majeures impliquant des vecteurs, avec des conséquences économiques importantes. Il y a tout de même un certain nombre d'agents pathogènes qui sont transmis par des vecteurs à l'animal, mais ils sont aussi transmis à l'homme. Ce qui était strictement animal était finalement assez rare, mais la fièvre catarrhale ovine est arrivée et il y a actuellement un certain nombre de zoonoses, des maladies communes à l'animal et à l'homme, qui sont en expansion et dont on peut craindre qu'elles continuent à se développer. C'est le cas de la fièvre de la vallée du Rift, qui est présente en France, à Mayotte, et dont nous pensons qu'elle pourrait arriver au Maghreb. De ce fait, il y a aussi chez les vétérinaires une prise de conscience. L'entomologie médicale et vétérinaire n'était pas enseignée dans les écoles vétérinaires ni dans les écoles d'agronomie, mais un effort a été fait ces dernières années pour y remédier. Il y a aussi un autre problème, c'est que nous sommes relativement démunis en lutte antivectorielle contre les vecteurs des maladies animales. On observe, on décrit, on essaye de comprendre et puis, finalement, on ne sait pas trop quoi faire de toutes ces informations pour être efficace. Et ça, j'espère que ça va changer bientôt, grâce aux conclusions de l'expertise collective sur la lutte antivectorielle.

NSS : On parle aussi en ce moment, justement par souci d'efficacité, de réunir une partie des chercheurs qui travaillent au CNRS en biologie avec ceux de l'Inserm dans un même institut. Est-ce que ça ne favoriserait pas finalement les collaborations, l'interdisciplinarité?

Didier Fontenille : Je pars du principe que, lorsque les chercheurs ont besoin de collaborations, ils ne se posent pas la question de l'appartenance institutionnelle. Regrouper les chercheurs en fonction de leurs compétences, ça les aide à travailler ensemble, mais il manquera toujours des compétences! Est-ce qu'on doit regrouper tous les biologistes d'un côté ou est-ce qu'on doit regrouper les collègues de différentes disciplines travaillant sur un même objet? Prenons un exemple caricatural, celui des systèmes vectoriels. Je vous ai expliqué que, pour étudier un système vectoriel, il fallait un anthropologue, un sociologue, un géographe, un économiste, des entomologistes, des médecins... Alors, pour être efficace, il faut créer un institut d'infectiologie dans lequel on mettra tous ces gens-là !

Je pense qu'il faut évidemment favoriser l'interdisciplinarité, mais il faut surtout que les gens soient ouverts. On peut avoir des mécanismes pour favoriser cette interdisciplinarité, en regroupant les chercheurs dans des structures, utiliser l'incitation financière, favoriser les interactions entre les personnes, ou avoir quelques aiguillons d'une manière ou d'une autre. Mais une boîte restera toujours une boîte, et il faut savoir en sortir !

NSS : Et pourtant, vous œuvrez largement pour la création d'un «Vectopole». En quoi est-ce différent?

Didier Fontenille : Le Vectopole est une plate-forme technique qui manque actuellement et c'est donc un petit peu différent. Nous avons besoin en France, et en particulier dans la région montpelliéraine où est regroupé le plus grand nombre de compétences françaises et probablement européennes autour des vecteurs, d'un d'outil technique. Ce Vectopole peut être le nucleus de quelque 
chose qui va au-delà, notamment devenir une structure permettant de conseiller en lutte antivectorielle, d'organiser des formations avec le ministère de la Santé et le ministère de l'Agriculture. Le Vectopole peut être une des composantes de ce que nous voudrions mettre en place : un centre national d'expertise sur les vecteurs de maladies humaines et animales. Ce sont des idées et des projets qui, de mon point de vue, répondent à un besoin national.

D'ailleurs, les problèmes auxquels est confrontée la France se posent au niveau européen, même si elle est un peu plus concernée à cause de ses départements et territoires d'outre-mer. Et puis la France, à cause de son histoire et de son passé colonial, a beaucoup d'échanges, par exemple avec l'Afrique noire. Il y a donc beaucoup plus d'importations d'agents pathogènes en France et beaucoup plus de Français de métropole qui vont être exposés (visites familiales, déplacements professionnels, tourisme...). Notre pays est particulièrement exposé à ce type de risque, mais les autres pays européens se posent la même question et il y a une volonté de mutualisation ou de réflexion commune au niveau européen avec l'European Center for Disease Control and Prevention.

J'ajouterai qu'il faut aussi s'intéresser à la question de la formation. Il y en a une à l'Institut Pasteur, qui est codirigée maintenant par l'IRD, et il y a des modules de formation au service de santé des armées dans lesquels l'IRD et l'Institut Pasteur interviennent. Mais il faut souligner qu'à la suite de l'enquête de Dominique Cuisance, nous avons créé un master international d'entomologie médicale et vétérinaire, qui est une formation complète de neuf mois, codirigée par 1'Université Montpellier 2 et 1'Université d'Abomey-Calavi au Bénin. C'est une formation à composante Sud, qu'on a voulue sur deux pays, voire deux continents différents. Dans ce master, interviennent des chercheurs de l'IRD, de l'Institut Pasteur, du Cirad et des intervenants internationaux : italiens, anglais, etc.

\section{Une discipline entre recherche et action...}

NSS : Puisque vous parlez de la nécessité de formation, de mettre en place une plate-forme technique, de créer un centre d'expertise sur les vecteurs, quelle est la nature des rapports que vous entretenez avec les acteurs plus opérationnels, qui exercent par exemple à la DRASS ou à l'EID?

Didier Fontenille : Ce n'est pas un lien évident. En France, nous avons des chercheurs, parfois de purs esprits, qui sont dans les instituts de recherche. Et puis, on a des opérateurs qui, eux, sont un peu les pieds dans la boue et luttent contre des vecteurs. Ceux-ci sont souvent des moustiques, mais peuvent être également des triatomes, par exemple pour la maladie de Chagas, des phlebotomes pour la leishmaniose ou, dans le domaine vétérinaire, des culicoïdes (qui sont des petits moucherons) pour la fièvre catarrhale ovine. Ce ne sont pas du tout les mêmes personnes qui font la lutte et qui travaillent sur l'objet insecte dans ces différents cas. C'est vrai que le lien était distendu, alors qu'il y a 50 ans, on faisait de l'entomologie médicale pour passer ensuite à l'opérationnel. Mais maintenant les liens sont à nouveau beaucoup plus forts, car les opérationnels sont mieux formés, certains ont fait des masters, parfois même des thèses, et le dialogue est plus facile. Et puis, malgré leur panoplie d'outils, les opérationnels se sont trouvés confrontés à des limites avec l'apparition de résistances aux insecticides. Il s'agit de mécanismes génétiques ou biochimiques très complexes, et il faut faire appel à la recherche pour les étudier. Il ne suffit donc pas de faire des tests, il faut savoir quels tests il faut faire et pourquoi, quel autre insecticide utiliser et comment mesurer l'efficacité de cet insecticide. Certaines agences opérationnelles, comme 1'EID de la région méditerranéenne ou le service de lutte antivectorielle du conseil général de la Martinique, ont eux-mêmes leurs propres structures de recherche. Ces structures de recherche sont en lien avec les instituts de recherche qui les aident. J'espère, en tout cas, que les structures que nous sommes en train de mettre en place en France, et en particulier le Vectopole, s'il se crée, ou le centre national d'expertise sur les vecteurs, aideront à l'instauration d'un réel dialogue entre recherche et action.

NSS : En retour, qu'est-ce que les acteurs peuvent aussi apporter aux chercheurs?

Didier Fontenille : Nous avons beaucoup de retours. Ce n'est pas nous qui mesurons l'efficacité de notre recherche, mais les opérateurs, même si nous pouvons participer à des mesures d'efficacité, des suivis d'indicateurs. Je vais prendre l'exemple le plus simple possible. Lorsqu'un nouvel insecticide est utilisé quelque part et que ça ne marche pas, les opérationnels se posent des questions et nous obligent à formuler de nouvelles questions de recherche. Certains gènes de la résistance aux insecticides ont été découverts chez des moustiques uniquement parce que les opérationnels disaient : "Il se passe quelque chose, ce qu'on fait ne marche pas! » En fait, les moustiques étaient devenus résistants à l'insecticide et ce sont des mécanismes génétiques nouveaux qui ont été mis en évidence par les chercheurs.

NSS : Vous faites état de la nécessité de faire collaborer une diversité d'acteurs, mais cela ne vous empêche-t-il pas d'atteindre parfois une réelle efficacité?

Didier Fontenille : La réponse est oui, évidemment. Nous pouvons avoir d'excellents outils qui parfois ne fonctionnent pas pour des raisons qui sont liées aux modes de gouvernance ou aux populations elles-mêmes. Par exemple, pour la démoustication, dans certains départements l'État fait tout, dans certains autres c'est la 
région qui est censée tout faire et dans d'autres encore des contrats sont passés avec des agences comme l'EID, voire des entreprises privées de démoustication. Cela ne facilite pas, de mon point de vue, l'efficacité. Je ne suis pas particulièrement jacobin, mais je pense que, dès que nous avons affaire à des problèmes de santé publique, c'est une question qui concerne l'État. Maintenant, les moustiques sont des populations locales et ceux qui sont au contact de ces populations, ce sont vraiment les collectivités locales. Il est donc évident qu'elles doivent être impliquées dans la lutte, et que l'État doit leur donner les moyens, pas seulement financiers mais aussi en termes de formations, etc. À un second niveau, nous nous heurtons à ce qu'on appelle la participation communautaire. Par exemple, on sait qu'il y a certains moustiques qui se développent à proximité des habitations, dans des gîtes créés par l'homme. Il serait stupide d'employer des insecticides, il vaut mieux détruire les gîtes. Dans d'autres cas, comme les leishmanioses forestières en Guyane, il n'y a qu'un seul moyen, c'est de se protéger avec des répulsifs. Pour Aedes albopictus, qui est présent à l'île de la Réunion, à Mayotte, en France métropolitaine, c'est aussi une solution efficace. Mais, parfois, l'implication des populations ou l'utilisation des insecticides ne suffisent pas, et il faudra trouver autre chose dans l'avenir.

\section{... en prise avec des questions de société}

NSS : D'autant que les groupes sociaux auxquels vous faites référence se posent des questions sur l'usage des insecticides et leur impact sur la santé humaine, ainsi que sur le respect des zones naturelles et de certaines espèces animales. Comment vous situez-vous sur cette question?

Didier Fontenille: Je vois les choses assez clairement. Un insecticide, c'est quelque chose qui est polluant ! Donc oui, il peut avoir des effets collatéraux adverses sur la faune non cible. Un insecticide peut aussi provoquer des intoxications ou des allergies chez les personnes qui le pulvérisent. C'est malheureusement l'outil que l'on devrait utiliser en dernier recours, mais le dernier recours arrive souvent. Mieux utiliser les insecticides implique une réflexion et une collaboration entre l'ensemble des acteurs : les chimistes qui connaissent les insecticides, les entomologistes qui vont travailler sur les insectes, les opérationnels qui vont les utiliser, les collègues des sciences humaines et sociales qui vont aussi apprécier les perceptions des populations concernées, les médecins qui vont suivre l'impact éventuel sur la santé et les écologues qui vont suivre l'impact sur le milieu. Donc, pour moi, il ne faut pas dire : «Les insecticides, il ne faut pas les utiliser ! » Bien sûr qu'il faut les utiliser quand il y en a besoin, toute la difficulté est de savoir à quel moment nous en avons besoin et pourquoi.

Nous ne pouvons pas actuellement nous en passer, même si nous essayons de trouver de nouvelles méthodes grâce à l'évolution des techniques. Il y a 30 ans, on travaillait déjà sur la lutte biologique, y compris à Montpellier, et cela n'a pas marché, sauf rare exception, comme par exemple en Chine, avec les poissons dans les rizières. Actuellement, en Thaillande, pour lutter contre le vecteur de la dengue, Aedes aegypti, est développé l'ensemencement avec des copépodes, qui sont des prédateurs des larves du moustique. Nous avons également dans notre équipe, à l'île de la Réunion, un programme de lâcher de moustiques mâles stériles dans la nature. Il faut savoir que seules les femelles de moustiques piquent et transmettent le virus. Donc, si nous lâchons des mâles, cela n'a pas d'implication épidémiologique, et les œufs pondus par les femelles qui ne sont fécondées qu'une seule fois sont stériles. C'est une méthode élégante pour diminuer les densités de population de moustiques. Maintenant, cela relève encore du domaine de la recherche, car nous ne savons pas exactement à quel moment les moustiques s'accouplent, si les mâles stérilisés sont aussi attractifs pour les femelles, s'ils ont autant de «sex-appeal » que les mâles sauvages, etc. De plus, il faut trouver des mécanismes de stérilisation, de distribution et d'élevage à grande échelle, parce qu'il va falloir lâcher des millions de moustiques mâles. Il y a également d'autres approches extrêmement intéressantes mais qui relèvent encore de la recherche, avec par exemple les moustiques transgéniques. Mais, là aussi, il faut mobiliser un ensemble de disciplines, dont la génétique, la génomique, l'écologie vectorielle et clairement les sciences sociales. Parce que transgénique, ça veut dire OGM et, si l'outil était efficace, est-ce que les populations françaises seraient prêtes à ce que des OGM volants soient lâchés dans la nature?

NSS : Justement, dans le cadre d'une problématique environnement-santé au sens le plus large du terme, estce que la difficulté n'est pas de mettre en place une prévention quand la société ne perçoit le moustique que comme une nuisance et non pas comme un vecteur de maladie?

Didier Fontenille : C'est une vraie question. Mais, à la limite, quand les habitants le perçoivent comme une nuisance, ils n'aiment pas la nuisance non plus. Je vais prendre un exemple un peu théorique en considérant que les moustiques constituent à $90 \%$ une nuisance et à $10 \%$ un vecteur. S'il n'y avait que les $10 \%$ de vecteur, les gens ne demanderaient pas de lutte antivectorielle parce qu'ils supporteraient, mais, grâce à la nuisance, ils l'acceptent. C'est exactement ce qui se passe en Afrique avec les moustiquaires imprégnées d'insecticide. Il arrive que les vecteurs du paludisme ne soient pas suffisamment abondants pour constituer une nuisance ; en revanche, quand ils transmettent le paludisme, les enfants meurent. Les populations acceptent beaucoup plus facilement les moustiquaires lorsqu'il y a une nuisance du vecteur. Maintenant, ce que j'appelle la prévention, c'est aussi de l'éducation, de la compréhension 
des perceptions et de la manière dont les gens peuvent conceptualiser ou s'approprier un peu l'histoire des vecteurs. C'est compliqué un vecteur, car ce n'est pas seulement un moustique, c'est aussi un insecte qui va prendre un agent pathogène, un virus ou un parasite, chez un hôte qui le porte, qui n'est pas obligatoirement malade, mais qui va le répliquer dans son corps pendant cinq, six ou une dizaine de jours ; et ensuite, à l'issue d'un repas de sang ultérieur, le transmettre à un nouvel hôte. Ce sont des concepts que tout le monde ne connaît pas, mais on peut probablement les faire acquérir par de l'éducation. Ce n'est pas mon métier, là encore, mais je pense que l'on pourrait envisager de parler davantage de la dengue, du paludisme et du Chikungunya dans les écoles des zones tropicales. En Martinique, c'est d'ailleurs ce qui se fait.

NSS : Et pour les pays tempérés, que faut-il faire à votre avis pour prendre en compte le risque de diffusion de certaines maladies vectorielles?

Didier Fontenille : Ce ne sont évidemment pas les mêmes écosystèmes, ni les mêmes variables climatiques, ni les mêmes systèmes de santé publique et vétérinaire, ni les mêmes systèmes de surveillance entre les pays du Sud et du Nord. Malgré tout, l'ensemble des composantes sont les mêmes, avec des variations selon les contextes. La situation est assez similaire entre les pays africains, sud-américains ou asiatiques. C'est le poids des différentes composantes qui va être différent, mais la plupart des maladies à transmission vectorielle sont aujourd'hui des maladies tropicales. Par contre, je me plais toujours à rappeler qu'il y a eu du paludisme en Scandinavie et qu'aux $\mathrm{XVII}^{\mathrm{e}}$ et $\mathrm{XVIII}^{\mathrm{e}}$ siècles, le paludisme tuait énormément dans les zones humides en France. La moitié des habitants de la ville d'Aigues-Mortes sont morts du paludisme il y a deux siècles. Simplement, dans les pays tempérés et par ailleurs un peu plus riches, il est beaucoup plus facile de lutter contre le paludisme. Or, la composante climatique et le niveau de développement font qu'il n'y a plus de paludisme en Europe. Mais la France, avec ses départements d'outre-mer, a quand même une composante tropicale forte ; et elle n'a jamais totalement perdu ses compétences en entomologie médicale, puisqu'il y a des collègues qui sont de bons spécialistes à l'île de la Réunion, en Martinique, en Guyane, en NouvelleCalédonie, etc. Maintenant, chose nouvelle, on prend conscience qu'il peut y avoir des problèmes également en France métropolitaine, avec le West Nile, la fièvre catarrhale ovine et un moustique invasif comme Aedes albopictus, vecteur potentiel du Chikungunya et de la dengue.
En ce qui me concerne, j'appartiens à l'IRD, dont les missions concernent plus particulièrement les pays en voie de développement. Comme nous avons une compétence tropicale, nous pouvons intervenir également dans les zones tropicales françaises, mais l'essentiel de nos activités doit se faire dans les pays du Sud qui en ont besoin. Je crois que tout ça est en redéfinition et je suis assez optimiste. Je pense que l'on va pouvoir mettre en place au niveau national, comme je l'ai déjà indiqué, une structure efficace d'expertise, de formation, voire de recherche, qui sera capable d'intervenir sur l'ensemble des domaines tropicaux ou tempérés.

NSS : L'entomologiste est donc aujourd'hui plutôt un homme heureux, parce qu'il se retrouve à la fois dans une dynamique de recherche fondamentale et appliquée et qu'il a un sentiment d'utilité sociale...

Didier Fontenille : Non, je n'emploierai peut-être pas le mot heureux, il n'est pas plus malheureux ou plus heureux qu'avant. L'entomologiste médical a évolué dans ses concepts, dans les disciplines qu'il mobilise. C'està-dire qu'on ne peut pas être complètement fermé sur une branche de la discipline, par exemple la systématique ou l'étude du génome ou au contraire les tests insecticides. Nous revenons d'une certaine manière à une vision plus naturaliste, mais en nous appropriant de nouveaux outils, comme la génétique, la génomique, l'utilisation d'images spatiales, les systèmes d'information géographique, etc. Du coup, nous travaillons aussi avec d'autres disciplines, comme les sciences humaines, la médecine depuis toujours et même, depuis peu, les mathématiques. Par exemple, nous développons beaucoup de modèles, et le travail fait avec Nicolas Ponçon sur l'évaluation du risque de réapparition du paludisme en Camargue relève de l'approche modélisatrice. En tout cas, pour un entomologiste, oui, je crois qu'on peut dire qu'il y a beaucoup de choses à faire encore dans l'avenir ; et c'est très stimulant intellectuellement, parce qu'il $\mathrm{y}$ a des vraies questions de recherche, soit très fondamentales, ciblées sur un point particulier, soit beaucoup plus systémiques, permettant d'associer différentes disciplines. Personnellement, ma sensibilité est plutôt d'avoir une vision d'ensemble. Or, du coup, la contrepartie, c'est qu'on a du mal à être très spécialisé dans l'ensemble de ces disciplines, et il faut arriver à trouver un juste équilibre. J'ai ma spécialité et ça ne m'empêche pas de m'intéresser au reste, et la meilleure façon d'y arriver est de travailler en équipe et d'accepter le dialogue entre disciplines et entre chercheurs et acteurs. 